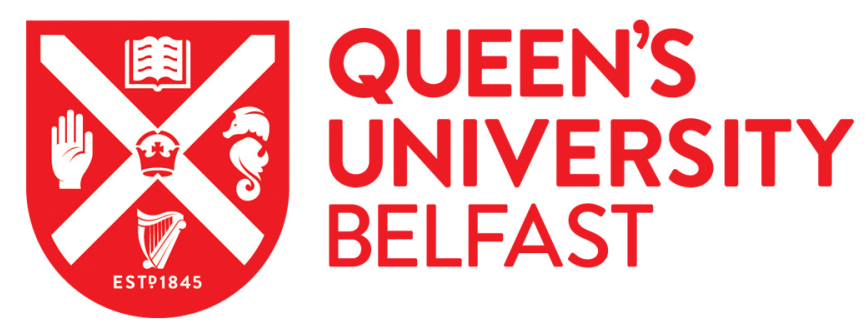

\title{
A polyhedral approach to the stability analysis and feedback stabilization of networked control systems
}

\begin{abstract}
Athanasopoulos, N., Bitsoris, G., \& Dritsas, L. (2011). A polyhedral approach to the stability analysis and feedback stabilization of networked control systems. In Proceedings of the 19th Mediterranean Conference on Control \& Automation Institute of Electrical and Electronics Engineers Inc..
\end{abstract}

https://doi.org/10.1109/MED.2011.5983214

Published in:

Proceedings of the 19th Mediterranean Conference on Control \& Automation

Document Version:

Peer reviewed version

Queen's University Belfast - Research Portal:

Link to publication record in Queen's University Belfast Research Portal

\section{General rights}

Copyright for the publications made accessible via the Queen's University Belfast Research Portal is retained by the author(s) and / or other copyright owners and it is a condition of accessing these publications that users recognise and abide by the legal requirements associated with these rights.

Take down policy

The Research Portal is Queen's institutional repository that provides access to Queen's research output. Every effort has been made to ensure that content in the Research Portal does not infringe any person's rights, or applicable UK laws. If you discover content in the Research Portal that you believe breaches copyright or violates any law, please contact openaccess@qub.ac.uk. 


\title{
A polyhedral approach to the stability analysis and feedback stabilization of networked control systems
}

\author{
Nikolaos Athanasopoulos, George Bitsoris, and Leonidas Dritsas
}

\begin{abstract}
In this note, the stability analysis and control design of Networked Control Systems (NCS) with bounded transmission delays, constant and unknown or time varying, is investigated using polyhedral Lyapunov functions. First, conditions guaranteeing stability are formulated for the equivalent augmented discrete-time system with structured uncertainties. Then, algebraic conditions leading to the computation of a linear state feedback controller for a NCS with bounded time varying delays are established. Both the stability analysis and synthesis problem are reduced to linear programming methods, a welcome consequence stemming from the choice of polyhedral Lyapunov functions.
\end{abstract}

\section{INTRODUCTION}

The major control challenge in analysis and synthesis of Networked Controlled System (NCS) is to face the problems due to the presence of uncertain network-induced delays [1], [2]. These delays stem from the information from sensor to controller and from controller to actuator. For the case of a discrete static feedback implemented with a period $h$, these delays can be lumped into a single term $\tau^{k}$, where $k$ refers to the sampling instant $k h$.

Significant effort has been invested in developing control methodologies to handle the network delay effect in NCSs (see surveys [1], [2]). Robust stability conditions for NCS with varying network-induced delays have been recently established using quadratic [3], [4] and non-quadratic Lyapunov functions [5]. In [6], LMI formulation is used for robust stability analysis and controller synthesis for NCS subject to uncertain time-varying delays bounded by a sampling period; the case of NCS with delays longer than one sampling period is presented in [7], [8]. By treating the uncertain NCS delay as a time-varying parameter uncertainty, sufficient conditions, expressed as LMIs, for the existence of a static stabilizing state feedback controller appear in [8], [9], [10]. Also, switched system approaches have recently been developed in [11], [12].

Most approaches use as analysis and synthesis tools either Lyapunov-Krasovskii functionals or quadratic Lyapunov functions. Recently, in [5], [13] polyhedral Lyapunov functions were used for the analysis and design of networked control systems that are described by ARMA models. The notion of the existence of invariant sets was also introduced for this class of systems, and stabilizing laws have been considered.

G. Bitsoris, N. Athanassopoulos and L. Dritsas are with the Control Systems Laboratory, University of Patras, Patras 26500, Greece. e-mail: nathanaseece.upatras.gr, bitsorisdece.upatras.gr, ldridotenet.gr
In this article the NCS are described by state equations with an augmented state vector composed of the states at two successive time instants and polyhedral Lyapunov functions are used. . The benefit of this approach is twofold: First, the choice of polyhedral Lyapunov functions along with the decomposition of the matrix exponential to its constituent matrices leads to the development of a methodology of stability analysis and control design based on the solution of a linear programming problems. Second, working in the augmented state space less conservative results are produced, since no extra properties guaranteeing the existence of positively invariant or contractive sets are imposed.

The article is organized as follows: Section II refers to the modelling aspects of NCS with varying transmission delays. In Section III, stability conditions for NCS systems are established. These conditions lead to the development of a method for the determination of the admissible delay range for systems controlled by linear state-feedback. In Section IV, the design problem of linear state-feedback controllers guaranteeing the stability for any delay belonging to a prespecified range is investigated. A numerical example concerning the control design of linear NCS is given in section $\mathrm{V}$.

Throughout this paper, capital letters denote real matrices and lower case letters denote column vectors or scalars. For two real vectors $x=\left[\begin{array}{llll}x_{1} & x_{2} & \ldots & x_{n}\end{array}\right]^{T}$ and $y=$ $\left[\begin{array}{llll}y_{1} & y_{2} & \ldots & y_{n}\end{array}\right]^{T} x<y \quad(x \leq y)$ is equivalent to $x_{i}<$ $y_{i} \quad\left(x_{i} \leq y_{i}\right)$ for $i=1,2, \ldots, n$. Similar notation holds for matrices. For real vectors $x$ and matrices $H$ with nonnegative entries we use the simplified notations $x \geq 0$ and $H \geq 0$ respectively. Given a real matrix $H=\left(h_{i j}\right), H^{+}=\left(h_{i j}^{+}\right)$ and $H^{-}=\left(h_{i j}^{-}\right)$are the nonnegative matrices with entries $h_{i j}^{+}=\max \left\{h_{i j}, 0\right\}$ and $h_{i j}^{-}=\max \left\{-h_{i j}, 0\right\}$ respectively. Thus, $H=H^{+}-H^{-}$. Finally, for a vector $x \in \mathbb{R}^{n},\|x\|$ denotes its infinity norm. $0_{n}$ and $1_{n}$ denote the $n \times n$ zero and identity matrices respectively.

\section{NCS-DYNAMICS}

The dynamics of the NCS under investigation is described by the combination of a continuous time linear time invariant plant with a discrete time controller and its configuration is shown in Figure 1. The sampling period $h$ is assumed to be constant and known, whereas both controller and actuator (including the zero-order-hold - $\mathrm{ZOH}$ ) are eventdriven devices in the sense that they update their outputs as soon as they receive a new sample. The state vector $x$ is sampled periodically, transmitted through the network, fed to the discrete-time controller which computes the control 
action and transmits it to the event-driven actuator after an uncertain delay. The plant receives this command after an uncertain delay $\tau^{k}$.

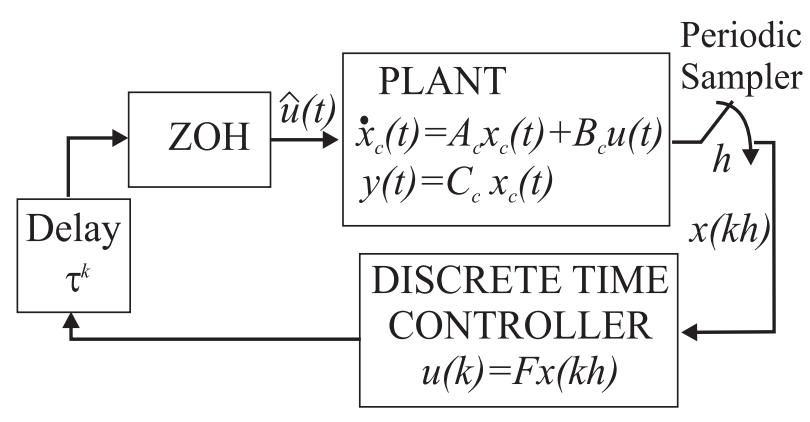

Fig. 1. NCS structural framework

In this article, the case of systems with less than one sampling period delay $\tau^{k}$, that is $\tau^{k}<h$, is considered. For the control architecture shown in Figure 1, the system dynamics is described below, for $t \in\left[k h+\tau^{k}, k h+h+\tau^{k+1}\right)$ :

$$
\begin{gathered}
\dot{x}(t)=A_{c} x(t)+B_{c} \hat{u}(t), \quad y(t)=C_{c} x(t), \\
\hat{u}(t)=u(k-1), \quad t \in\left[k h-h+\tau^{k-1}, k h+\tau^{k}\right)
\end{gathered}
$$

where $A_{c} \in \mathbb{R}^{n \times n}, B_{c} \in \mathbb{R}^{n \times m}, C_{c} \in \mathbb{R}^{s \times n}$. Signal $\hat{u}(t)$ represents the most recent control action presented to the eventdriven actuator at the time instance $t$ within a sampling period, that is within the time interval $[k h, k h+h)$. The total delay within the $k$ th sampling period is denoted by $\tau^{h}$ and is assumed upper bounded, i.e. $0 \leq \tau_{\min }<\tau^{k} \leq \tau_{\max }=h$. In general it is a time varying and uncertain quantity, reflecting the nature of the network involved, the network load, etc.

Since the actuation time instances are not equidistant, the piecewise constant control action $\hat{u}(t)$ experiences a "jump" at the uncertain time instance $k h+\tau^{k}$. Thus, it is not in general possible to treat the ensuing NCS in a standard sampled-data or time-delayed setting [2], [15]. However, the exact discretization of (1),(2) is straightforward and is given by [14]:

$$
x(k+1)=\Phi x(k)+\Gamma_{0}\left(\tau^{k}\right) u(k)+\Gamma_{1}\left(\tau^{k}\right) u(k-1)
$$

where $\Phi=\exp \left(A_{c} h\right)$ and

$$
\begin{aligned}
& \Gamma_{0}\left(\tau^{k}\right)=\int_{0}^{h-\tau^{k}} \exp \left(A_{c} t\right) B_{c} d t \\
& \Gamma_{1}\left(\tau^{k}\right)=-\Gamma_{0}\left(\tau^{k}\right)+\int_{0}^{h} \exp \left(A_{c} t\right) B_{c} d t .
\end{aligned}
$$

The uncertain delay can always be written as $\tau^{k}=\tau^{\circ}+\tau_{\Delta}^{k}$ with $\tau^{\circ}$ denoting the selected nominal value, $\tau^{o} \in\left[\tau_{\min }, \tau_{\max }\right]$. Consequently, matrices $\Gamma_{0}\left(\tau^{k}\right), \Gamma_{1}\left(\tau^{k}\right)$ can be decomposed into constant and known nominal parts $\Gamma_{0}\left(\tau^{\circ}\right), \Gamma_{1}\left(\tau^{\circ}\right)$ and uncertain, although bounded, parts $\Delta \Gamma_{0}\left(\tau^{k}, \tau^{\circ}\right), \Delta \Gamma_{1}\left(\tau^{k}, \tau^{\circ}\right)$, that is

$$
\Gamma_{i}\left(\tau^{k}\right) \triangleq \Gamma_{i}\left(\tau^{\circ}\right)+\Delta \Gamma_{i}\left(\tau^{k}, \tau^{\circ}\right) \quad i=0,1
$$

where

$$
\begin{gathered}
\Gamma_{0}\left(\tau^{\circ}\right)=\int_{0}^{h-\tau^{\circ}} \exp \left(A_{c} t\right) B_{c} d t, \quad \Gamma_{1}\left(\tau^{\circ}\right)=\int_{h-\tau^{\circ}}^{h} \exp \left(A_{c} t\right) B_{c} d t \\
\Delta \Gamma_{1}\left(\tau^{k}, \tau^{\circ}\right)=\int_{h-\tau^{k}}^{h-\tau^{\circ}} \exp \left(A_{c} t\right) B_{c} d t=-\Delta \Gamma_{0}\left(\tau^{k}, \tau^{\circ}\right)
\end{gathered}
$$

System (3) can thus be equivalently written in the form

$$
\begin{aligned}
x(k+1)= & \Phi x(k)+\left[\Gamma_{0}\left(\tau^{\circ}\right)+\Delta \Gamma_{0}\left(\tau^{k}, \tau^{\circ}\right)\right] u(k)+ \\
& +\left[\Gamma_{1}\left(\tau^{\circ}\right)+\Delta \Gamma_{1}\left(\tau^{k}, \tau^{\circ}\right)\right] u(k-1) .
\end{aligned}
$$

When a discrete-time linear state feedback law $u(k)=F x(k)$ is applied, the closed-loop dynamics becomes

$$
x(k+1)=\left[\Phi+\Gamma_{0}\left(\tau^{k}\right) F\right] x(k)+\Gamma_{1}\left(\tau^{k}\right) F x(k-1)
$$

or

$$
\begin{aligned}
x(k+1)= & \Phi x(k)+\left[\Gamma_{0}\left(\tau^{\circ}\right)+\Delta \Gamma_{0}\left(\tau^{k}, \tau^{\circ}\right)\right] F x(k)+ \\
& +\left[\Gamma_{1}\left(\tau^{\circ}\right)+\Delta \Gamma_{1}\left(\tau^{k}, \tau^{\circ}\right)\right] F x(k-1) .
\end{aligned}
$$

\section{STABILITY ANALYSIS OF NCS}

Equation (8) describing a delayed discrete-time system can be equivalently written in state equations form in the augmented state space. Setting

$$
z(k) \triangleq\left[x(k)^{T} \quad x(k-1)^{T}\right]^{T},
$$

we establish the equivalent description for the closed-loop dynamics

$$
z(k+1)=\left[\begin{array}{cc}
\Phi+\Gamma_{0}\left(\tau^{k}\right) F & \Gamma_{1}\left(\tau^{k}\right) F \\
I_{n} & 0_{n}
\end{array}\right] z(k) .
$$

Using (8), equation (9) can be equivalently written as

$$
\begin{gathered}
z(k+1)=\left[\begin{array}{cc}
\Phi+\Gamma_{0}\left(\tau^{\circ}\right) F & \Gamma_{1}\left(\tau^{\circ}\right) F \\
I_{n} & 0_{n}
\end{array}\right] z(k)+ \\
+\left[\begin{array}{cc}
\Delta \Gamma_{0}\left(\tau^{k}, \tau^{\circ}\right) F & \Delta \Gamma_{1}\left(\tau^{k}, \tau^{\circ}\right) F \\
0_{n} & 0_{n}
\end{array}\right] z(k) .
\end{gathered}
$$

Thus, system (9) is described by a state equation of the form

$$
z(k+1)=A_{d}^{\circ} z(k)+\Delta A_{d}\left(\tau^{k}, \tau^{\circ}\right) z(k)
$$

and is decomposed into a nominal system part characterized by matrix $A_{d}^{\circ} \in R^{2 n \times 2 n}$

$$
A_{d}^{\circ}=\left[\begin{array}{cc}
\Phi+\Gamma_{0}\left(\tau^{\circ}\right) F & \Gamma_{1}\left(\tau^{\circ}\right) F \\
I_{n} & 0_{n}
\end{array}\right]
$$

which is a function of $\tau^{\circ}$, the selected gain $F$ and an uncertain part $\Delta A_{d}\left(\tau^{k}, \tau^{\circ}\right) \in \mathbb{R}^{2 n \times 2 n}$

$$
\Delta A_{d}\left(\tau^{k}, \tau^{\circ}\right)=\left[\begin{array}{cc}
\Delta \Gamma_{0}\left(\tau^{k}, \tau^{\circ}\right) F & \Delta \Gamma_{1}\left(\tau^{k}, \tau^{\circ}\right) F \\
0_{n} & 0_{n}
\end{array}\right]
$$

being clearly a function of the uncertain delay $\tau^{k}$ and of the selected gain $F$.

Nominal matrix $A_{d}^{\circ}$ is time invariant and can be computed given the continuous time dynamics, the sampling period 
and the static feedback gain. Furthermore, using the relation $\Delta \Gamma_{1}\left(\tau^{k}\right)=-\Delta \Gamma_{0}\left(\tau^{k}\right)$, the uncertain "perturbation" matrix $\Delta A_{d}$ can be expressed as

$$
\Delta A_{d}\left(\tau^{k}, \tau^{\circ}\right)=\left[\begin{array}{cc}
-\Delta \Gamma_{1}\left(\tau^{k}, \tau^{\circ}\right) F & \Delta \Gamma_{1}\left(\tau^{k}, \tau^{\circ}\right) F \\
0_{n} & 0_{n}
\end{array}\right] .
$$

In the sequel, for notation brevity, we omit the dependence of $\Delta A_{d}\left(\tau^{k}, \tau^{\circ}\right)$ and $\Delta \Gamma_{1}\left(\tau^{k}, \tau^{\circ}\right)$ on $\tau^{k}$ and $\tau^{\circ}$ and we use the notations $\Delta A_{d}$ and $\Delta \Gamma_{1}$ respectively.

Inhere, the following issue is addressed: Given a controller $u(k)=\left[\begin{array}{ll}F & 0\end{array}\right]^{T} z(k)$ which stabilizes the nominally delayed closed-loop system, that is such that $\left|\operatorname{eig}\left(A_{d}^{\circ}\right)\right|<1$, what is it to be expected in terms of stability when the same control law is used for the uncertain discrete-time system in (10)? Moreover, since the varying network delay is reflected into the uncertain matrices $\Delta A_{d}$ and $\Delta \Gamma_{1}$, is it possible to quantify the answer in terms of a "delay range" for which asymptotic stability of the closed-loop system is guaranteed?

Stability of uncertain varying systems

$$
x(k+1)=A(\tau) x(k)
$$

with $\tau$ belonging to a set $\tau \in\left[\tau_{\min }, \tau_{\max }\right]$ is guaranteed if there exists a common Lyapunov function for all members $S_{\tau}$ of the family of time invariant systems (11) with $\tau \in\left[\tau_{\min }, \tau_{\max }\right]$. Such stability conditions are given in the following lemma:

Lemma 1: If for a matrix $G \in \mathbb{R}^{p \times 2 n}, \max _{1 \leq j \leq p}\left\{(G x)_{j}>\right.$ 0\} $\forall x \neq 0$, a vector $w \in \mathbb{R}^{p}$ with positive components and any $\tau \in\left[\tau_{\min }, \tau_{\max }\right]$ there exist a positive real number $\varepsilon<1$ and matrices $H(\tau) \in \mathbb{R}^{p \times p}$ satisfying the relations

$$
\begin{gathered}
G A(\tau)=H(\tau) G \\
H(\tau) w \leq \varepsilon w \\
H\left(\tau^{k}\right) \geq 0
\end{gathered}
$$

then system (11) is asymptotically stable.

This Lemma is an extension to uncertain systems of a basic result developed for time-invariant systems [17]. Relations (12) and (13) are the necessary and sufficient conditions for the positive definite scalar function

$$
v(z) \triangleq \max _{1 \leq i \leq 2 n}\left\{\frac{(G z)_{i}}{w_{i}}\right\}
$$

to be a Lyapunov function of all systems (11) with $\tau \in$ $\left[\tau_{\min }, \tau_{\max }\right]$. Moreover, $v(x(k+1)) \leq \varepsilon v(x(k))$, that is, $\varepsilon$ is a measure of the exponential rate convergence of state $x(k)$ to the equilibrium $x=0$.

Application of this result to system (9) provides stability conditions that relate the network delay with the feedback controller gains:

Theorem 2: If for a matrix $G \in \mathbb{R}^{p \times 2 n}, \max _{1 \leq j \leq p}\left\{(G x)_{j}>\right.$ 0\} $\forall x \neq 0$, a vector $w \in \mathbb{R}^{p}$ with positive components and any $\tau^{k} \in\left[\tau_{\min }, \tau_{\max }\right]$ there exist a positive real number $\varepsilon<1$ and matrices $H\left(\tau^{k}\right) \in \mathbb{R}^{p \times p}$ satisfying the relations

$$
G\left[\begin{array}{cc}
\Phi+\Gamma_{0}\left(\tau^{k}\right) F & \Gamma_{1}\left(\tau^{k}\right) F \\
I_{n} & 0_{n}
\end{array}\right]=H\left(\tau^{k}\right) G
$$

$$
H\left(\tau^{k}\right) \geq 0
$$

then system (8) is asymptotically stable.

A direct consequence of this result is the following corollary where conditions guaranteeing the asymptotic stability of NCS with a fixed delay $\tau^{*}$ are established.

Corollary 3: If there exist a matrix $G \in$ $\mathbb{R}^{p \times 2 n}, \max _{1 \leq j \leq p}\left\{(G x)_{j}>0\right\} \forall x \neq 0$, a vector $w \in \mathbb{R}^{p}$ with positive components, a nonnegative real number $\varepsilon\left(\tau^{*}\right)<1$ and a matrix $H^{\circ}\left(\tau^{*}\right) \in \mathbb{R}^{p \times p}$ such that

$$
\begin{gathered}
G\left[\begin{array}{cc}
\Phi+\Gamma_{0}\left(\tau^{*}\right) F & \Gamma_{1}\left(\tau^{*}\right) F \\
I_{n} & 0_{n}
\end{array}\right]=H\left(\tau^{*}\right) G \\
H\left(\tau^{*}\right) w<\varepsilon\left(\tau^{*}\right) w \\
H\left(\tau^{*}\right) \geq 0
\end{gathered}
$$

then system (8) with fixed delay $\tau^{*}$ is asymptotically stable.

Remark 4: The above relations can be used to determine the delay bounds ensuring stability for a given linear state feedback gain when constant and unknown delays are present. In particular, for a certain gain $F$, the following linear programming problems

$$
\min _{H\left(\tau^{*}\right), \varepsilon\left(\tau^{*}\right)}\left\{\varepsilon\left(\tau^{*}\right)\right\}
$$

subject to (18)-(20) are solved for different values of the delay $\tau^{k}$. If the optimal value $\varepsilon\left(\tau^{*}\right)$ is less than one, asymptotic stability is ensured for system (9) for the delay $\tau^{k}=\tau^{*}$.

\section{Robust State FEedback Stabilization}

Although conditions of Theorem 2 guarantee asymptotic stability for a constant and unknown delay $\tau^{*}$, they cannot be applied in a straightforward manner to the case of uncertain time varying delays. This is due to the fact that in this case matrices $\Gamma_{0}\left(\tau^{k}\right)$ and $\Gamma_{1}\left(\tau^{k}\right)$ depend on an unknown and time-varying parameter $\tau^{k}$ and as a result this is also true for the undetermined matrix $H\left(\tau^{k}\right)$. A similar problem is faced when LMI methods or Model Predictive Control approaches to stabilization of linear parameter varying systems are used. Another approach consists in modelling the delay-induced terms $\Delta \Gamma_{0}\left(\tau^{k}, \tau^{\circ}\right)$ and $\Delta \Gamma_{1}\left(\tau^{k}, \tau^{\circ}\right)$ using a polytopic approximation. In a recent work [18] an approach, based on convex polytopic inclusions, to the construction of discrete-time models of linear systems with time-varying input delays has been developed. In this article, the uncertain matrices $\Delta \Gamma_{0}\left(\tau^{k}, \tau^{\circ}\right)$ and $\Delta \Gamma_{1}\left(\tau^{k}, \tau^{\circ}\right)$ are expressed in terms of the constituent matrices of the open loop matrix $A_{c}$. This decomposition, combined with the use of polyhedral Lyapunov functions, leads to conditions guaranteeing asymptotic stability of NCS controlled by linear state feedback.

The exponential $\exp \left(A_{c} t\right)$ of a matrix $A_{c}$ can always be written as

$$
\exp \left(A_{c} t\right)=a_{1}(t) Z_{1}+a_{2}(t) Z_{2}+\ldots+a_{n}(t) Z_{n}
$$


where $Z_{i} \in \mathbb{R}^{n \times n} i=1,2, \ldots, n$ are real matrices, the so called "constituent matrices", and $a_{i}(t) i=1,2, \ldots, n$ are real functions corresponding to the eigenvalues $\lambda_{i}$ of matrix $A_{c}$. To each real eigenvalue $\lambda$ with multiplicity $r$, there correspond functions $a_{q}(t)$ given by relations $a_{q}(t)=t^{q} e^{\lambda t}$ $q=0,1, \ldots, r-1$ and to each pair of conjugate complex eigenvalues $\lambda=\sigma \pm j \omega$ of multiplicity $r$ there correspond pairs of functions $a_{c q}(t)=t^{q} e^{\sigma t} \cos (\omega t)$ and $a_{s q}(t)=t^{q} e^{\sigma t} \sin (\omega t)$ $q=0,1, \ldots, r-1$. Therefore,

$$
\begin{gathered}
\Delta \Gamma_{1}\left(\tau^{k}, \tau^{\circ}\right)=\int_{h-\tau^{k}}^{h-\tau^{\circ}} \exp \left(A_{c} t\right) B_{c} d t= \\
=\sum_{i=1}^{n} \int_{h-\tau^{k}}^{h-\tau^{\circ}} a_{i}(t) d t Z_{i} B_{c}=\sum_{i=1}^{n} c_{i}\left(\tau^{k}\right) Z_{i} B_{c} .
\end{gathered}
$$

where $c_{i}\left(\tau^{k}\right)$ are integrals of the form

$$
c_{i}\left(\tau^{k}\right)=\int_{h-\tau^{k}}^{h-\tau^{\circ}} t^{q} e^{\lambda t} d t
$$

in the case of a real eigenvalue $\lambda$, or

$$
c_{i}\left(\tau^{k}\right)=\int_{h-\tau^{k}}^{h-\tau^{\circ}} t^{q} e^{\sigma t} \cos (\omega t) d t
$$

and

$$
c_{i}\left(\tau^{k}\right)=\int_{h-\tau^{k}}^{h-\tau^{\circ}} t^{q} e^{\sigma t} \sin (\omega t) d t
$$

in the case of a pair of conjugate complex eigenvalues $\lambda=$ $\sigma \pm j \omega$. We are in a position to state the following:

Theorem 5: If for a matrix $G \in \mathbb{R}^{p \times 2 n}, \max _{1 \leq j \leq p}\left\{(G x)_{j}>\right.$ $0 \forall x \neq 0$ and a vector $w \in \mathbb{R}^{p}$ with positive components there exist real $p \times p$ matrices $H^{\circ}, H_{Z_{1}}, H_{Z_{2}}, \ldots, H_{Z_{n}}$, and a real number $\varepsilon<1$ such that

$$
\begin{gathered}
G\left[\begin{array}{cc}
\Phi+\Gamma_{0}\left(\tau^{\circ}\right) F & \Gamma_{1}\left(\tau^{\circ}\right) F \\
I_{n} & 0_{n}
\end{array}\right]=H^{\circ} G \\
G\left[\begin{array}{cc}
-Z_{i} B F & Z_{i} B F \\
0_{n} & 0_{n}
\end{array}\right]=H_{Z_{i}} G \quad i=1,2, \ldots, n \\
H^{\circ}+\sum_{i=1}^{n} c_{i}\left(\tau^{k}\right) H_{Z_{i}} \geq 0 \quad \forall \tau^{k} \in\left[\tau_{\min }, \tau_{\max }\right] \\
\left(H^{\circ}+\sum_{i=1}^{n} c_{i}\left(\tau^{k}\right) H_{Z_{i}}\right) w \leq \varepsilon w \quad \forall \tau^{k} \in\left[\tau_{\min }, \tau_{\max }\right]
\end{gathered}
$$

then the equilibrium $x=0$ of system (8) is asymptotically stable.

Proof: From (23) and (25) it follows that

$$
G\left[\begin{array}{cc}
\Delta \Gamma_{0}\left(\tau^{k}, \tau^{\circ}\right) F & \Delta \Gamma_{1}\left(\tau^{k}, \tau^{\circ}\right) F \\
0_{n} & 0_{n}
\end{array}\right]=
$$

$$
\begin{aligned}
& =G\left[\begin{array}{cc}
-\sum_{i=1}^{n} c_{i}\left(\tau^{k}\right) Z_{i} B_{c} F & \sum_{i=1}^{n} c_{i}\left(\tau^{k}\right) Z_{i} B_{c} F \\
0_{n} & 0_{n}
\end{array}\right]= \\
& =\sum_{i=1}^{n} c_{i}\left(\tau^{k}\right) G\left[\begin{array}{cc}
-Z_{i} B_{c} F & Z_{i} B_{c} F \\
0_{n} & 0_{n}
\end{array}\right]= \\
& =\sum_{i=1}^{n} c_{i}\left(\tau^{k}\right) H_{Z_{i}} G
\end{aligned}
$$

Thus,

$$
\begin{gathered}
G\left[\begin{array}{cc}
\Phi+\Gamma_{0}\left(\tau^{k}\right) F & \Gamma_{1}\left(\tau^{k}\right) F \\
I_{n} & 0_{n}
\end{array}\right]= \\
=G\left[\begin{array}{cc}
\Phi+\Gamma_{0}\left(\tau^{\circ}\right) F & \Gamma_{1}\left(\tau^{\circ}\right) F \\
I_{n} & 0_{n}
\end{array}\right]+ \\
G\left[\begin{array}{cc}
-\Delta \Gamma_{1}\left(\tau^{k}, \tau^{\circ}\right) F & \Delta \Gamma_{1}\left(\tau^{k}, \tau^{\circ}\right) F \\
0_{n} & 0_{n}
\end{array}\right]= \\
=H^{\circ} G+\sum_{i=1}^{n} c_{i}\left(\tau^{k}\right) H_{Z_{i}} G=\left(H^{\circ}+\sum_{i=1}^{n} c_{i}\left(\tau^{k}\right) H_{Z_{i}}\right) G .
\end{gathered}
$$

Taking into account (26) and (27) we conclude that hypotheses of Theorem 1 are satisfied for

$$
H\left(\tau^{k}\right)=H^{\circ}+\sum_{i=1}^{n} c_{i}\left(\tau^{k}\right) H_{Z_{i}}
$$

Therefore the equilibrium $x=0$ of system (8) is asymptotically stable.

Remark 6: Since $\tau^{\circ} \in\left[\tau_{\min }, \tau_{\max }\right]$, there exists a $\tau^{k} \in$ $\left[\tau_{\min }, \tau_{\max }\right]$ such that $c_{i}\left(\tau^{k}\right)=0$. Then, for conditions (26) and (27) to be satisfied it is necessary that $H^{\circ} \geq 0$ and $H^{\circ} w<w$. These relations together with (24) guarantee the asymptotic stability of the positive invariance of set $P(G, w)$ w.r.t. to the nominal augmented system

$$
z(k+1)=\left[\begin{array}{cc}
\Phi+\Gamma_{0}\left(\tau^{\circ}\right) F & \Gamma_{1}\left(\tau^{\circ}\right) F \\
I_{n} & 0_{n}
\end{array}\right] z(k)
$$

Therefore, conditions (24)-(27) of Theorem 4 can be partitioned into two groups: The first one, composed of relations (24), $H^{\circ} \geq 0$ and $H^{\circ} w<w$ refers to the nominal system and guarantee the asymptotic stability of the equilibrium $x=0$ and the positive invariance of set $P(G, w)$ w.r.t. this system. These conditions do not depend on the delay time $\tau^{k}$. The second group is composed of relations (25)-(27) and define implicitly the range of admissible delays $\tau^{k}$ for which the asymptotic stability of the equilibrium $x=0$ and the positive invariance of set $P(G, w)$ is preserved.

The next step, is to replace conditions (26) and (27) by relations non depending on the delay time $\tau^{k}$ but only on the range $\left[\tau_{\min }, \tau_{\max }\right]$. Setting

$$
H_{Z_{i}}=H_{Z_{i}}^{+}-H_{Z_{i}}^{-}
$$

relations (26) and (27) can be written as

$$
H^{\circ}+\sum_{i=1}^{n} c_{i}\left(\tau^{k}\right) H_{Z_{i}}^{+}-\sum_{i=1}^{n} c_{i}\left(\tau^{k}\right) H_{Z_{i}}^{-} \geq 0
$$

$$
\left(H^{\circ}+\sum_{i=1}^{n} c_{i}\left(\tau^{k}\right) H_{Z_{i}}^{-}-\sum_{i=1}^{n} c_{i}\left(\tau^{k}\right) H_{Z_{i}}^{+}\right) w \leq \varepsilon w,
$$


$\forall \tau^{k} \in\left[\tau_{\min }, \tau_{\max }\right]$. Let

$$
c_{i \max } \triangleq \max _{\tau_{\min } \leq \tau^{k} \leq \tau_{\max }}\left\{c_{i}\left(\tau^{k}\right)\right\} .
$$

Then

$$
\begin{gathered}
H^{\circ}+\sum_{i=1}^{n} c_{i}\left(\tau^{k}\right) H_{Z_{i}}= \\
=H^{\circ}+\sum_{i=1}^{n} c_{i}\left(\tau^{k}\right) H_{Z_{i}}^{+}-\sum_{i=1}^{n} c_{i}\left(\tau^{k}\right) H_{Z_{i}}^{-} 0 \geq \\
\geq H^{\circ}-\sum_{i=1}^{n} \max \left\{c_{i \max }, 0\right\} H_{Z_{i}}^{+}+\sum_{i=1}^{n} \min \left\{c_{i \min }, 0\right\} H_{Z_{i}}^{-}
\end{gathered}
$$

$\forall \tau^{k} \in\left[\tau_{\min }, \tau_{\max }\right]$ because, by definition, $H_{Z_{i}}^{+} \geq 0$ and $H_{Z_{i}}^{-} \geq 0$. Therefore, condition (26) is satisfied if

$$
H^{\circ}-\sum_{i=1}^{n} \max \left\{c_{i \max }, 0\right\} H_{Z_{i}}^{+}+\sum_{i=1}^{n} \min \left\{c_{i \min }, 0\right\} H_{Z_{i}}^{-} \geq 0
$$

Using similar arguments we can also prove that condition (27) is satisfied if

$$
\left(H^{\circ}+\sum_{i=1}^{n} \min \left\{c_{i \min }, 0\right\} H_{Z_{i}}^{+}-\sum_{i=1}^{n} \max \left\{c_{i \max }, 0\right\} H_{Z_{i}}^{-}\right) w \leq \varepsilon w .
$$

We are now in a position to establish the following result where no conditions depending on the uncertain or time varying delay time $\tau^{k}$ are present.

Theorem 7: If for a matrix $G \in \mathbb{R}^{p \times n}, \max _{1 \leq j \leq p}\left\{(G x)_{j}\right\}>0$ $\forall x \neq 0$ and a vector $w \in \mathbb{R}^{p}$ with positive components, there exist nonnegative matrices $H^{\circ}, H_{Z_{1}}^{+}, H_{Z_{1}}^{-}, H_{Z_{2}}^{+}, H_{Z_{2}}^{-} \ldots, H_{Z_{n}}^{+}, H_{Z_{n}}^{-}$ such that

$$
\begin{gathered}
G\left[\begin{array}{cc}
\Phi+\Gamma_{0}\left(\tau^{\circ}\right) F & \Gamma_{1}\left(\tau^{\circ}\right) F \\
I_{n} & 0_{n}
\end{array}\right]=H^{\circ} G \\
G\left[\begin{array}{cc}
-Z_{i} B F & Z_{i} B F \\
0_{n} & 0_{n}
\end{array}\right]=H_{Z_{i}} G \quad i=1,2, \ldots, n \\
H^{\circ}-\sum_{i=1}^{n} \max \left\{c_{i \max }, 0\right\} H_{Z_{i}}^{+}+\sum_{i=1}^{n} \min \left\{c_{i \min }, 0\right\} H_{Z_{i}}^{-} \geq 0 \\
\left(H^{\circ}+\sum_{i=1}^{n} \min \left\{c_{i \min }, 0\right\} H_{Z_{i}}^{+}-\sum_{i=1}^{n} \max \left\{c_{i \max }, 0\right\} H_{Z_{i}}^{-}\right) w \leq \varepsilon w
\end{gathered}
$$

then the equilibrium $x=0$ of system (8) is asymptotically stable and set $P(G, w)$ is positively invariant for any time varying delay $\tau^{k} \in\left[\tau_{\min }, \tau_{\max }\right]$.

Conditions (32) and (33) can be simplified in the case when the open-loop matrix $A_{c}$ has real eigenvalues. In this case, if $\tau^{\circ}=\tau_{\min }=0$, then parameters $c_{i}\left(\tau^{k}\right)$ are nonnegative and increasing in $\left[\tau_{\min }, \tau_{\max }\right]$. This implies

$$
c_{i \max } \triangleq c_{i}\left(\tau_{\max }\right)=\int_{h-\tau_{\max }}^{h-\tau^{\circ}} t^{q} e^{\lambda t} d t \geq 0
$$

and

$$
c_{i \min } \triangleq c_{i}\left(\tau_{\min }\right)=\int_{h-\tau_{\min }}^{h-\tau^{\circ}} t^{q} e^{\lambda t} d t=0
$$

Therefore, in this case, conditions (32) and (33) can be equivalently expressed as

$$
\begin{gathered}
H_{0}^{\circ}-\sum_{i=1}^{n} c_{i}\left(\tau_{\max }\right) H_{Z_{i}}^{+} \geq 0 \\
\left(H^{o}-\sum_{i=1}^{n} c_{i}\left(\tau_{\max }\right) H_{Z_{i}}^{-}\right) w \leq \varepsilon w
\end{gathered}
$$

Thus, we establish the following corollary of Theorem 6:

Corollary 8: If the open-loop- matrix $A_{c}$ has real eigenvalues and for a matrix $G \in \mathbb{R}^{p \times n}, \max _{1 \leq j \leq p}\left\{(G x)_{j}\right\}>0 \forall x \neq 0$ and a vector $w \in \mathbb{R}^{p}$ with positive components, there exist nonnegative matrices $H^{\circ}, H_{Z_{1}}^{+}, H_{Z_{1}}^{-}, H_{Z_{2}}^{+}, H_{Z_{2}}^{-} \ldots, H_{Z_{n}}^{+}, H_{Z_{n}}^{-}$such that

$$
\begin{gathered}
G\left[\begin{array}{cc}
\Phi+\Gamma_{0}\left(\tau^{\circ}\right) F & \Gamma_{1}\left(\tau^{\circ}\right) F \\
I_{n} & 0_{n}
\end{array}\right]=H^{\circ} G \\
G\left[\begin{array}{cc}
-Z_{i} B F & Z_{i} B F \\
0_{n} & 0_{n}
\end{array}\right]=H_{Z_{i}} G \quad i=1,2, \ldots, n \\
H^{\circ}-\sum_{i=1}^{n} c_{i}\left(\tau_{\max }\right) H_{Z_{i}}^{+} \geq 0 \\
\left(H^{\circ}-\sum_{i=1}^{n} c_{i}\left(\tau_{\max }\right) H_{Z_{i}}^{-}\right) w \leq \varepsilon w
\end{gathered}
$$

then the equilibrium $x=0$ of system (8) is asymptotically stable and set $P(G, w)$ is positively invariant for any time varying delay $\tau^{k} \in\left[\tau_{\min }, \tau_{\max }\right]$.

A solution to relations (30)-(33) can be obtained by defining an optimization problem having these relations as linear constraints. Thus, a state-feedback control law $u(k)=F x(k)$ that stabilizes the NCS for any delay time $\tau^{k} \in\left[\tau_{\min }, \tau_{\max }\right]$ is determined by solving the linear programming problem

$$
\min _{\varepsilon, F, H^{\circ}, H_{1}^{\circ}, H_{Z_{i}}^{+}, H_{Z_{i}}^{-}}\{\varepsilon\}
$$

under linear constraints

$$
\begin{gathered}
G\left[\begin{array}{cc}
\Phi+\Gamma_{0}\left(\tau^{\circ}\right) F & \Gamma_{1}\left(\tau^{\circ}\right) F \\
I_{n} & 0_{n}
\end{array}\right]=H^{\circ} G \\
G\left[\begin{array}{cc}
-Z_{i} B F & Z_{i} B F \\
0_{n} & 0_{n}
\end{array}\right]=H_{Z_{i}} G \quad i=1,2, \ldots, n \\
H^{\circ}-\sum_{i=1}^{n} \max \left\{c_{i \max }, 0\right\} H_{Z_{i}}^{+}+\sum_{i=1}^{n} \min \left\{c_{i \min }, 0\right\} H_{Z_{i}}^{-} \geq 0 \\
\left(H^{\circ}+\sum_{i=1}^{n} \min \left\{c_{i \min }, 0\right\} H_{Z_{i}}^{+}-\sum_{i=1}^{n} \max \left\{c_{i \max }, 0\right\} H_{Z_{i}}^{-}\right) w \leq \varepsilon w \\
H^{\circ}, H_{Z_{1}}^{+}, H_{Z_{1}}^{-}, H_{Z_{2}}^{+}, H_{Z_{2}}^{-} \ldots, H_{Z_{n}}^{+}, H_{Z_{n}}^{-} \geq 0_{p} .
\end{gathered}
$$

If the optimal value of parameter $\varepsilon$ is less than one, then the corresponding control law $u(k)=F x(k)$ is a solution to the problem under consideration. It should be emphasized that, according to Theorem 2, minimization of parameter $\varepsilon$ results in improved transient behavior, because parameter $\varepsilon$ is a measure of the exponential convergence of the state to the equilibrium of the augmented delayed system. 


\section{NumERICAL EXAMPLE}

In this example, the control synthesis problem is investigated for the benchmark system [4] with system matrices

$$
A_{c}=\left[\begin{array}{cc}
0 & 1 \\
0 & -0.1
\end{array}\right], \quad B_{c}=\left[\begin{array}{c}
0 \\
0.1
\end{array}\right]
$$
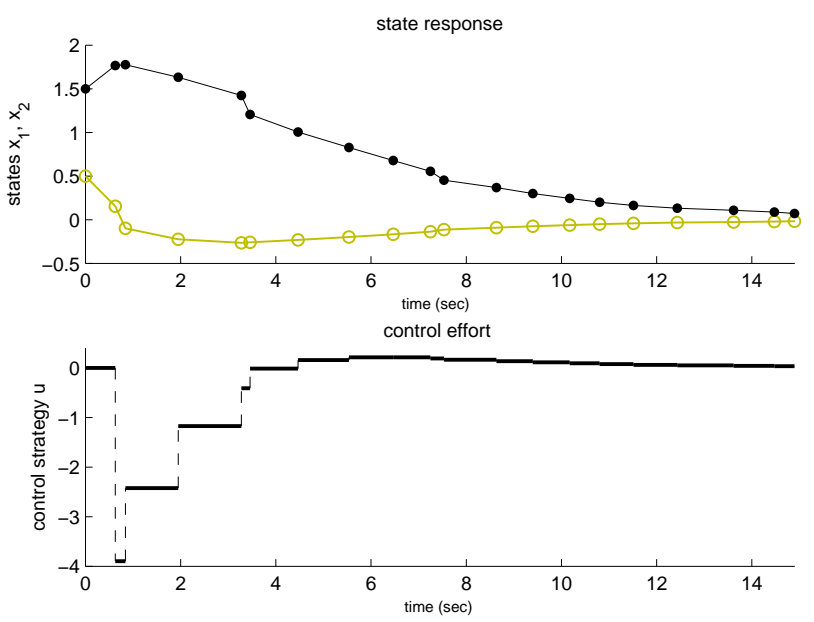

Fig. 2. State response and control effort for the closed loop NCs, with initial conditions $x(-1)=x(0)=\left[\begin{array}{ll}1.5 & 0.5\end{array}\right]^{T}\left(x_{1}\right.$ denoted by "." while $x_{2}$ is denoted by " $o$ ".)

The sampling period is chosen to be $h=0.82 \mathrm{sec}$. The control design problem consists in finding a linear state feedback control law $u(k)=F x(k)$ such that the resulting closed-loop NCS is asymptotically stable for time varying uncertain delays $\tau^{k} \in\left[\begin{array}{ll}\tau_{\min } & \tau_{\max }\end{array}\right]$, where $\tau_{\min }=0, \tau_{\max }=$ $h=0.82 \mathrm{sec}$. In this setting, we choose the nominal value of the delay $\tau^{o}$ to be equal to the minimum value, that is $\tau^{o}=$ $\tau_{\text {min }}=0$. Since all eigenvalues of the open-loop continuoustime system are real, that is $\lambda_{1}=0, \lambda_{2}=-0.1$, we can use the results stated in Corollary 8 . Solving the linear programming problem (39)-(43) with performance index (38), the optimal solution of the optimal value is $\varepsilon^{*}=0.79$. Thus, a stabilizing control law is computed with feedback gain

$$
F=\left[\begin{array}{ll}
-0.94 & -4.98
\end{array}\right] \text {. }
$$

In Fig. 2, the state response of the closed-loop system as well as the corresponding control strategy are shown, for initial condition $x(-1)=x(0)=\left[\begin{array}{ll}1.5 & 0.5\end{array}\right]^{T}$.

\section{CONCLUSIONS}

The main novelty of the approach presented in this article is, first, the use of polyhedral Lyapunov functions for the stability analysis and control design of networked control systems and, second, the decomposition of the matrix exponential to its constituent matrices in order to alleviate the uncertainty factor depending on the time varying delay $\tau^{k}$. It has been shown that the computational effort for producing the results is small since only linear optimization problems are involved. It is also worth mentioning that when we are only interested in stability with no additional requirements of existence of positively invariant sets, less conservative results are obtained.

\section{REFERENCES}

[1] T.C. Yang, "Networked control system: a brief survey," Control Theory and Applications, IEE Proceedings, vol. 153, pp. 403-4122, July 2006.

[2] J. Hespanha, P. Naghshtabrizi, and Y. Xu, "A Survey of Recent Results in Networked Control Systems," Proceedings of IEEE - Special Issue on Technology of Networked Control Systems, vol. 95, pp. 138-162, Jan. 2007.

[3] L. Dritsas and A. Tzes, "Robust stability analysis of networked systems with varying delays," International Journal of Control, vol. 82, pp. 2347-2355, Dec. 2009.

[4] L. Dritsas and A. Tzes, "Robust Stability Bounds for Networked Systems with Varying Delays," in Proceedings of the 17th Mediterranean Conference on Control and Automation, (Thessaloniki, Greece), pp. 778-783, June 2009.

[5] G. Bitsoris, N. Athanasopoulos, and L. Dritsas, "Feedback Stabilization of Networked Control Systems," in Proceedings of the 18th IEEE International Conference on Control Applications (Saint Petersburg, Russia), pp. 1-6, July 2009.

[6] M. Cloosterman, N. Wouw, M. Heemels, and H. Nijmeijer, "Robust Stability of Networked Control Systems with Time-varying Networkinduced Delays," in Proc. of the 45th IEEE Conference on Decision and Control, (San Diego, CA, U.S.A.), pp. 4980-4985, Dec. 2006.

[7] M. Cloosterman, N. Wouw, M. Heemels, and H. Nijmeijer, "Stability of Networked Control Systems with Large Delays," in Proc. of the 46th IEEE Conference on Decision and Control, (New Orleans, LA, U.S.A.), pp. 5017-5022, Dec. 2007.

[8] Y. Pan, H. Marquez, and T. Chen, "Stabilization of Remote Control Systems with Unknown Time Varying Delays by LMI Techniques," International Journal of Control, vol. 79, pp. 752-763, July 2006.

[9] D. Yue, Q. Han, and P. Chen, "State feedback controller design of networked control systems," IEEE Transactions on Circuits and Systems II, vol. 51, no. 11, pp. 640-644, 2004.

[10] D. Yue, Q. Han, and J. Lam, "Network-based robust $H_{\infty}$ control of systems with uncertainty," Automatica, vol. 41, no. 2, pp. 999-1007, 2005.

[11] L. Hetel, J. Daafouz, and C. Iung, "Stabilization of Arbitrary Switched Linear Systems With Unknown Time-Varying Delays," IEEE Trans. on Automatic Control, vol. 51, no. 10, pp. 1668-1674, 2006.

[12] L. Hetel, J. Daafouz, and C. Iung, "Analysis and control of LTI and switched systems in digital loops via an event-based modelling," International Journal of Control, vol. 81, no. 7, pp. 1125-1138, 2007.

[13] G. Bitsoris, N. Athanasopoulos, and L. Dritsas, "Stability, Positive Invariance and Design of Constrained State Feedback Regulators for Networked Control Systems", submitted to International Journal of Control, 2011.

[14] L. Dritsas and A. Tzes, "Modeling Approaches and Robust Stability Conditions for Networked Controlled Systems with Uncertain Delays," in Proceedings of the 17th IFAC World Congress, (Seoul, Korea), pp. 6353-6358, July 2008.

[15] P. Naghshtabrizi, J. Hespanha, and A. Teel, "On the robust stability and stabilization of sampled-data systems: A hybrid system approach," in Proceedings of the 45th IEEE Conference on Decision and Control, (San Diego, CA, U.S.A.), pp. 4873-4878, Dec. 2006.

[16] N. Athanasopoulos, and G. Bitsoris, "Invariant set computation for constrained uncertain discrete-time systems", in Proceedings of the 49th IEEE Conference on Decision and Control, Atlanta, GA, USA, pp. 5227-5232, 2010.

[17] G. Bitsoris and M. Vassilaki, "Design Techniques of constrained regulators for discrete-time systems," Control and Dynamic Systems, vol. 56, pp. 1-49, 1993. C.T. Leondes Editor.

[18] R.H. Gielen, S. Olaru, M. Lazar,W.P.M.H. Heemels, N. van de Wouw, and S.-I. Niculescu, "On polytopic inclusions as a modeling framework for systems with time-varying delays," Automatica, vol. 46, pp. 615619, Mar. 2010. 